\section{Diagnosis of exocrine pancreatic insufficiency in cystic fibrosis by use of fluorescein dilaurate test}

Sir,

Professor Cuschieri and colleagues suggest that use of fluorescein dilaurate in an oral pancreatic function test may, by detecting different levels of pancreatic function, differentiate between patients with cystic fibrosis who do not require exogenous enzyme supplementation and those who do.' The authors also propose the fluorescein dilaurate test as a screening test for pancreatic insufficiency to reduce the number of false positive diagnoses of cystic fibrosis. Examination of their data leaves me sceptical of these claims made on behalf of the test, for the following reasons.

(1) The implicit suggestion in this paper, that positive correlation of fluorescein dilaurate test result with faecal chymotrypsin value indicates this test to be semi-quantitative in assessing pancreatic function, is unsupported; I know of no evidence that faecal chymotrypsin correlates well with more direct measurements of exocrine state. Had faecal chymotrypsin values from the control group been presented, I suspect the overall correlation between faecal chymotrypsin and fluorescein dilaurate test result would have been very poor.

(2) The authors chose a cut off point of 20 for the fluorescein dilaurate test result, above which level gross pancreatic hypofunction was held to be excluded. A single patient with cystic fibrosis had a urinary fluorescein dilaurate value above this figure but also experienced no change in bowel habit once enzyme supplements were withdrawn. If, on further investigation, this patient was confirmed to have pancreatic sufficiency, the fluorescein dilaurate test as a screening test for pancreatic disease would seem to be no more sensitive than a clinical history of steatorrhoea. If, on the other hand, this same patient was shown indeed to have pancreatic insufficiency the fluorescein dilaurate test would have proved to be less sensitive than a simple measurement of faecal chymotrypsin, low in all the group with cystic fibrosis.

(3) We are told little about the controls other than that they were healthy. The fluorescein dilaurate test results show a very wide scatter $(13 \cdot 2-62 \cdot 2)$, with three of the 15 giving values of 20 or below-that is, by the authors' criteria, indication for further investigation of pancreatic function. Used as a screening test for pancreatic disease, $20 \%$ false positive results give a specificity of only $80 \%$. I wonder if the faecal chymotrypsin values in controls might have proved considerably more specific?

A simple, non-invasive test of pancreatic function with both high sensitivity (positivity in disease) and high specificity (negativity in absence of disease) together with the ability to delineate degrees of functional capacity must be considered yet to be found.
Reference
' Cumming JGR, Forsyth JS, Boyd EJS, Frost GJ, Cuschieri A.
Diagnosis of exocrine pancreatic insufficiency in cystic fibrosis
by use of fluorescein dilaurate test. Arch Dis Child 1986;61:
$573-5$.
J PUNTIS
Institute of Child Health,
Birmingham B16 $8 E T$

\section{Recurrent neural tube defects, risk factors and vitamins}

Sir,

Wild and her colleagues conclude that the highly significant difference in recurrence rates of neural tube defects between mothers who took periconceptual vitamins and those who did not, in the study reported by Smithells and his colleagues, was due to vitamin supplementation. ${ }^{1}$ They argue this on the basis that no significant confounding was found with certain factors such as social class or immediately prior miscarriage.

There are two problems with the paper. The first problem is that statistical re-analysis of the existing data cannot overcome bias that may have been introduced through patients presenting themselves and complying with supplementation being, in any case, at a relatively low risk of having a neural tube defect pregnancy. Allowing for known confounding factors cannot, of course, avoid bias from unknown ones. Although the authors conclude that bias from unknown confounding factors is unlikely to be of importance, we believe that in this particular instance it may have been critical. Most cases of neural tube defects, including recurrences, cannot be predicted by known means, indicating that the main risk factors are still unrecognised and could therefore exert a substantial confounding effect. Also adjusting for certain variables that might be confounding factors, but in fact were not, and then showing that adjustment for these variables did not alter the magnitude of the association is uninformative. It does not alter the strength of the evidence concerning whether the association was causal or due to bias.

The second problem relates to social class, and this has been the subject of previous publications. ${ }^{2-5}$ One of us, with Professor Polani, has shown that some bias was introduced because, as expected, women in the vitamin supplementation group who took extra vitamins tended to be of higher social class than women who did not; they were also, by virtue of their social class, less likely to have a further pregnancy associated with a neural tube defect. We used data from the first cohort of pregnancies in the vitamin supplementation study conducted by Smithells and his colleagues. In a letter replying to our paper they said that if data from both the first and second cohorts were aggregated no social class confounding was evident (the conclusion that Wild and her colleagues reach again in their present paper). Our response to the letter still 
applies-namely, that for there to be no bias in the first and second cohorts combined, women of relatively high social class in the second cohort must have had a high risk of neural tube defect recurrence, a surprising result that is contrary to knowledge of the epidemiology of the disorder. Indeed, the opposite effects in the two cohorts are extremely unlikely to have arisen by chance. The relative risk of having a neural tube defect pregnancy for 'low' social class women compared with 'high' social class women was 2.98 in the first cohort and 0.19 in the second, after allowing for supplementation $(p=0.003)$. This apparently inconsistent result between the two parts of the study needs to be explained before it can be concluded that, on average, social class was not related to neural tube defects and so could not have biased the results.

\section{References \\ 1 Wild J, Read AP, Sheppard S, et al. Recurrent neural tube defects, risk factors and vitamins. Arch Dis Child 1986;61: 440-4. \\ 2 Smithells RW, Seller MJ, Harris R, et al. Further experience of vitamin supplementation for prevention of neural tube defect recurrences. Lancet 1983;i:1027-31. \\ 3 Wald NJ, Polani PE. Neural-tube defects and vitamins: the need for a randomized clinical trial. $\mathrm{Br} J$ Obstet Gynaecol 1984;91:516-23. \\ ${ }^{4}$ Smithells RW, Sheppard S, Wild J, et al. Neural-tube defects and vitamins: the need for a randomized clinical trial. $\mathrm{Br} \mathrm{J}$ Obstet Gynaecol 1985;92:185-6. \\ 5 Wald NJ, Polani PE. Neural-tube defects and vitamins: the need for a randomized clinical trial. $B r \quad J$ Obstet Gynaecol 1985;92:187-8.}

Nicholas Wald and Simon G Thompson St Bartholomew's Hospital Medical College, London ECIM 6BM

Drs Wild and Sheppard and Professor Smithells comment: The points raised by Professor Wald and Dr Thompson have been raised and responded to in the papers they cite and elsewhere. Further reiteration would not be helpful, but we must again refute their inaccurate statement that our observations on social class and recurrence of neural tube defects are "contrary to knowledge of the epidemiology of the disorder'. We are aware of only one published study of recurrence of neural tube defects by social class. ${ }^{1}$ This showed a lower recurrence rate in classes I + II than in III + IV + V, but the difference was not significant.

Wald and Thompson believe that 'the main risk factors (for neural tube defects) are still unrecognised' but are curiously reluctant to consider, on the evidence, that vitamins might be one of them. We quite understand, however, that Professor Wald is not open to persuasion in this matter and we wish him all success in his own study.

\footnotetext{
Reference

1 Nevin NC, Johnston WP, Merrett JD. Influence of social class on the risk of recurrence of anencephalus and spina bifida. Dev Med Child Neurol 1981;23:155-9.
}

\section{Plasma zinc concentrations in iron supplemented low birthweight infants}

Sir,

We were interested to read the recent paper by Salvioli et al on plasma zinc concentrations in iron supplemented infants. ${ }^{1}$ It is indeed an interesting question whether low birthweight babies fed an iron fortified formula need additional iron. The authors conclude that they do, but we wonder whether a difference in mean haemoglobin of $9 \mathrm{~g} / \mathrm{l}$ is important and whether a haemoglobin $<110 \mathrm{~g} / \mathrm{l}$ signifies anaemia in the first year of life. The study was not a randomised trial; one group received iron supplements for more than five months, whereas the other group had not received iron supplements for three months but could have previously for up to nine months. The authors also address the question of whether iron supplements influence plasma zinc concentrations and imply that plasma zinc concentrations reflect zinc state or nutrition. This is an incorrect assumption as plasma zinc is highly influenced by recent dietary intake, stress, the metabolic state of the patient, postnatal age, and a diurnal variation. It is not mentioned when, in relation to feeds or iron supplements, the blood samples were taken.

A ratio of inorganic iron:zinc greater than 2 has repeatedly been shown to influence zinc absorption. It is likely that the zinc absorption in their infants, who received iron and zinc in a 5:1 ratio, would have been reduced. Whether this is important, however, remains to be shown. We feel that it should be made clear that the authors were not studying zinc state or zinc absorption but only plasma zinc concentrations. The importance of their findings is unclear and surely does not reassure us that iron supplements do not influence zinc metabolism.

\section{Reference}

' Salvioli GP, Faldella G, Alessandroni R, Lanari M, Benfenati L. Plasma zinc concentrations in iron supplemented low birthweight infants. Arch Dis Child 1986;61:346-8.

\section{Karen Simmer and $\mathrm{R} P \mathrm{H}$ Thompson St Thomas's Hospital, London SEI $7 E H$}

\section{Dr Salvioli comments:}

The only purpose of our study was to determine whether administration of high supplemental iron to low birthweight infants might result in zinc deficiency, as manifested by a decrease in plasma zinc.

According to Yip et al, although serum zinc is not a reliable indicator of zinc nutrition in individuals, it probably provides useful information for groups of subjects. ${ }^{1}$

Thus the equivalent plasma zinc concentrations found in two groups of low birthweight infants, comparable in all but the amount of iron supplement, reassures us that long 